\title{
Effect of Spatial Modulation on Pulse Width for Ultrashort Laser Pulse during Small-Scale Self-Focusing
}

\author{
Nian $\mathrm{Wang}^{1}$, Xiquan $\mathrm{Fu}^{1 *}$ \\ ${ }^{1}$ Key laboratory for Micro/Nano Optoelectronic Devices of Ministry of Education \\ ${ }^{1}$ Department of Computer Science and Electronic Engineering, Hunan University, Changsha \\ 410082, China
}

Keywords: ultrashort laser pulse, small-scale self-focusing, growth point, pulse width.

\begin{abstract}
Small-scale self-focusing (SSSF) causes the distribution of spatial intensity to become asymmetrical. Spatial distribution would generate new growth point during SSSF. We measure the spatial-temporal evolution for ultrashort laser pulse with different truncation parameter of knife-edge during SSSF. Our results show that the new growth point is increasingly far away from center of beam and its intensity increases with decrease of truncation parameter. Meanwhile, the pulse width at the growth point becomes narrower. We find that our experimental results are in good agreement with an approximate theoretical analysis.
\end{abstract}

\section{Introduction}

It is a known fact that spatial diffraction is the main reason for the initial intensity modulation. Initial intensity modulation is inevitable due to the confined aperture size of various transmitting devices in the laser system [1] and the existence of noise during the process of transmission [2]. Spatial diffraction can cause the intensity of the laser pulse to produce small-scale modulation and then the intensity distribution becomes asymmetrical [3]. This situation is easily leading to generating SSSF in nonlinear case [4]. SSSF leaded to off-axis energy moving toward the peak of the pulse and the laser pulse compressed in both space and time, which caused the peak intensity augmenting $[5,6]$. The increase of peak intensity caused self-phase modulation (SPM) to enhance, which leaded to generating new frequency components [7, 8]. If the peak intensity of the laser pulse continually increased, then the combination of the SPM-induced up-chirp and normal group velocity dispersion acted to push the energy away from the peak position, thus initiated the breaking up of the pulse $[9,10]$. It is generally accepted that SSSF is described in terms of the modulation instability (MI) model that was first proposed by Bespalov and Talanov [11]. Then the B-T theory was verified experimentally by Campillo [12] and Bliss [13]. MI is one of the most elementary effects, which associated with wave propagation in nonlinear medium. The gain spectrum of MI leads to amplification of sidebands, which breaks up the otherwise uniform wave front and generates fine localized structures.

In high power laser, SSSF is one of the most fundamental phenomena in nonlinear optics. It is the major factor limiting the maximum power available from a high power solid-state laser $[14,15]$. So SSSF has traditionally been attracted more attention and had extensity research in experimental and theoretical [16-17]. Many studies are based on different peak power [18, 19], the form of spatial modulation [20-22], and different initial chirped [23]. Another, the spatial-temporal evolution for laser pulse is also the main research domain. The direct time-resolved observation of the propagation of intensity femtosecond laser pulses experiencing SF, beam filamentation is investigated extensively [24-26]. Previously, we have observed the evolution of spatial modulation in the process of SSSF of a beam, the impact of relaxation effect in the media on spatial-temporal instability [27, 28]. However, these studies mostly concentrate on the spatial evolution and temporal evolution independent. They only describe the spatial-temporal coupling phenomenon through experiment. They didn't explain the detailed reason for the temporal evolution during the SSSF.

In this paper, the propagation regularity of knife-edge diffraction is systematically expounded. We find that the new growth point is different with the truncation parameter. The spatial intensity of 
growth point strengthens with the decrease of truncation parameter. Then, we show the temporal evolution of the new growth point. The increase of spatial intensity for the new growth point will lead to pulse width compression. At last, approximate theoretical analysis is used to verify our experimental results.

The paper is organized as follows. In section 2, the experimental setup of knife-edge diffraction is expounded. In section 3, the spatial-temporal evolution with the different diffraction parameter for ultrashort laser pulse during SSSF is analyzed. Section 4 presented the detailed theoretical analysis for temporal evolution.

\section{Experimental setup}

A schematic overview of our experiment setup is shown in Fig.1. We use an amplified Ti: sapphire laser system (Coherent Libra S, $\lambda_{0}=800 \mathrm{~nm}$ ) delivering pulses with bandwidth of $12 \mathrm{~nm}$, single pulse energy of $1 \mathrm{~mJ}$, duration $\tau_{0}=100 \mathrm{fs}$ at $1 \mathrm{kHz}$ repetition rate. The beam is split into two beams by a beam splitter, BS1, one is used as a pump beam and the other as a probe beam. The probe beam passes through a variable optical delay line before it is directed to knife-edge. The direction of the probe beam is changed by a rotating mirror in front of the knife-edge, according that the pump beam can be scanned by the probe beam when M5 is rotated. The spatial and temporal scanning resolution can be controlled by adjusting the position of knife-edge. A glass cuvette with $5 \mathrm{~cm}$ path length is filled with carbon disulfide $\left(\mathrm{CS}_{2}\right) . \mathrm{CS}_{2}$ is chosen as the nonlinear medium because of its strong Kerr nonlinearity. The pump beam is directed to the $\mathrm{CS}_{2}$ reflected by M3. A variable attenuator, $\mathrm{A} 1$, is used to adjust the input power of the pump beam. A knife-edge with thickness of $0.1 \mathrm{~mm}$ in front of $\mathrm{CS}_{2}$ is used to generate initial diffraction modulation. The evolution of spatial-temporal of the pump pulse can be monitored by two synchronized high-resolution charge-coupled device CCD cameras (pixel size 1,280×1,024, resolution $6.7 \mu \mathrm{m} \times 6.7 \mu \mathrm{m}$ ). In order to measure the spatial evolution of the pump beam in the front surface of BBO crystal, a part of the pump beam is imaged onto CCD1 reflected by a beam splitter, BS2. It follows that the optical path from the beam splitter, BS2, to BBO crystal is equal to from BS2 to CCD1. A sum-frequency beam generated in the $\mathrm{BBO}$ nonlinear crystal under non-collinear interaction of two beams is collected with a CCD2. So we can indirectly measure the temporal evolution of the pump pulses by the sum-frequency beam based on the cross-correlation principle. The adjustable attenuators, A2 and A3, are custom-designed to protect CCD cameras from damage by high power laser beams.

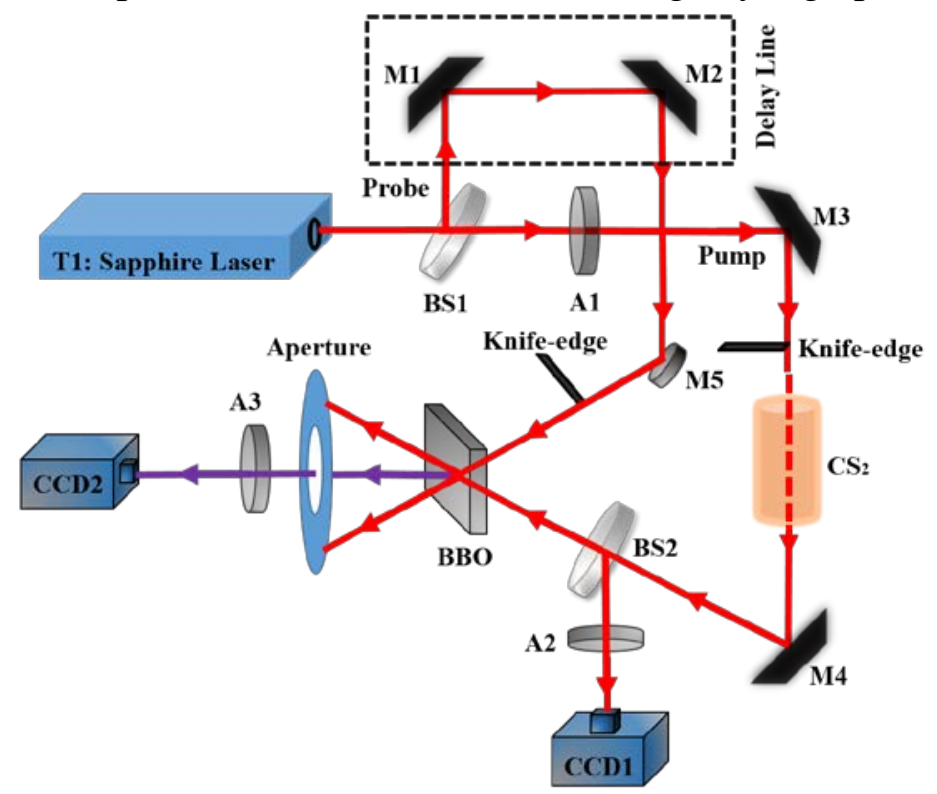

Fig. 1 Experimental setup: M1-M5 plane mirrors, A1-A3 attenuators, BS1-BS2 beam splitters. We are interested in the nonlinear diffraction from a knife-edge: 


$$
\psi(x, 0)= \begin{cases}1 & x>a \\ 0 & x<a\end{cases}
$$

We specify that $\alpha=a / w_{0}$ is the truncation parameter of knife-edge where $a$ is the $\mathrm{x}$-coordinate of knife-edge, $w_{0}$ is waist radius of beam. During the experiment, the below experiment parameters are used: $n_{0}=1.63, \mathrm{n}_{2}=3.5 \times 10^{-15} \mathrm{~cm}^{2} / \mathrm{W}, \beta_{2}=1.54 \times 10^{-25} \mathrm{~s}^{2} / \mathrm{m}$. In experiment, different results of spatial modulation for beam be acquired by adjusting the value of $\mathrm{x}$-coordinate of knife-edge. The different truncation parameter means the energy of mean which pass the knife-edge is different

\section{Experimental results}

Fig. 2 shows the spatial evolution of ultrashort laser pulse with different truncation parameter during SSSF. It can be observed from Fig. 2 that the new modulation growth point of SSSF appears in P1. When the truncation parameter is big, the influence of nonlinear effect is far less than the diffraction effect and the diffraction modulation plays a major role. So the SSSF at P1 is not obvious and the intensity of diffraction modulation peak P2 is more than P1 when the truncation parameter is 1.5 in Fig. 2(a). The nonlinear effect enhances with the decrease of truncation parameter, which causes the modulation growth of SSSF at P1 zone to strengthen. It is evident from Fig. 2(a)-2(d) that the intensity of the new growth point P1 increases rapidly with the decrease of truncation parameter. When the truncation parameter decreased to 0.9 , the intensity of the growth area $\mathrm{P} 1$ reaches maximum. As the truncation parameter continually decrease, the effect of SSSF cannot increase because the energy of beam is finite.
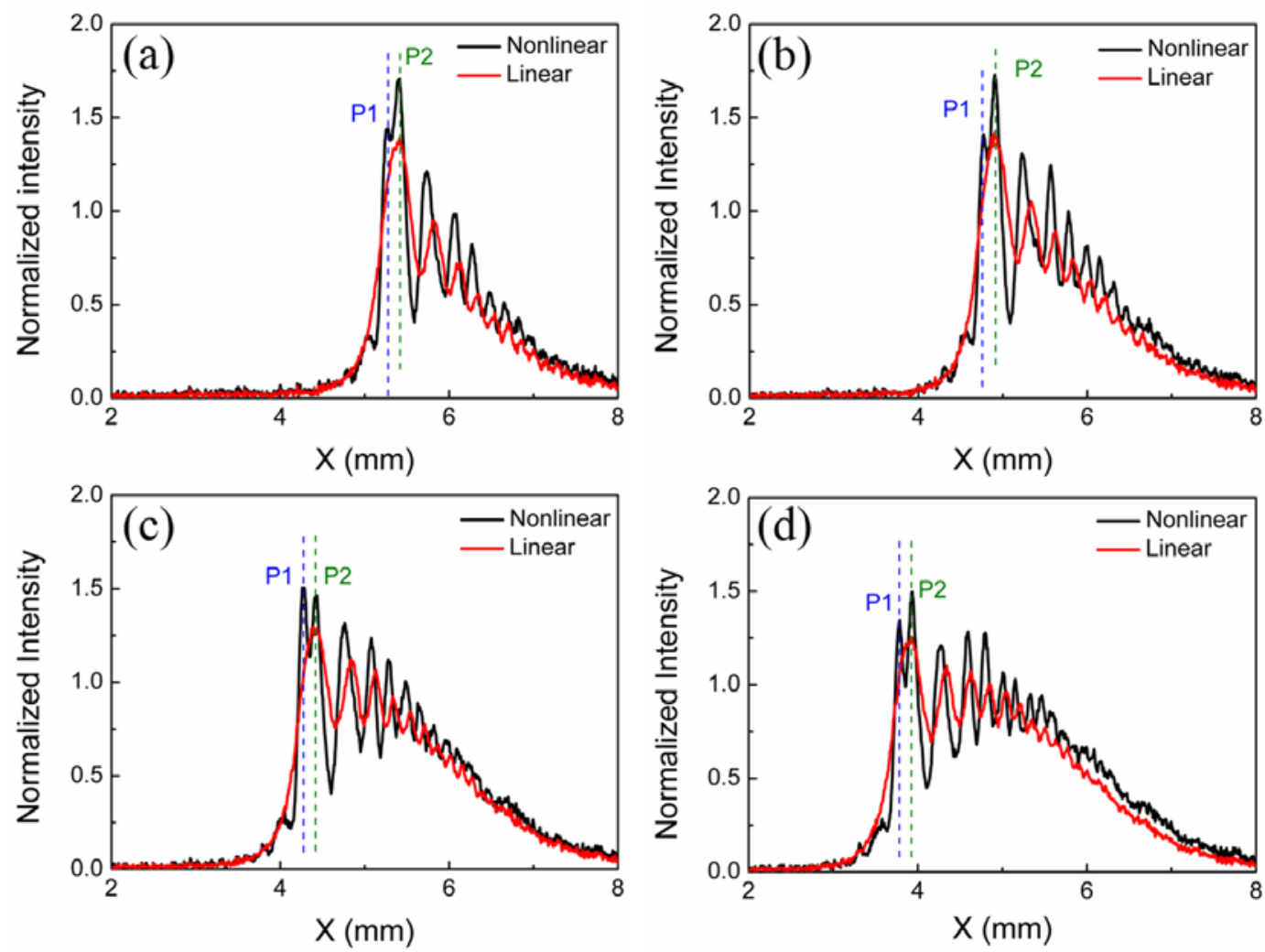

Fig. 2 The spatial distribution of SSSF varies with truncation parameter. (a)-(d) The truncation parameter is 1.5, 1.4, 1.3 and 0.9 respectively.

In fact, the energy of the pump beam which passes through the knife-edge increase with the decrease of truncation parameter. As the energy increased, the peak power of the pump beam increases. Meanwhile, the effect of SPM strengthen, which leads to the energy of laser pulse moving towards the modulation growth of SSSF. Fig. 3 indicates that the peak power reduces with the increment of the truncation parameter. The changeable of peak power would modify the position 
of the fastest growth frequency according to the property of MI [29].

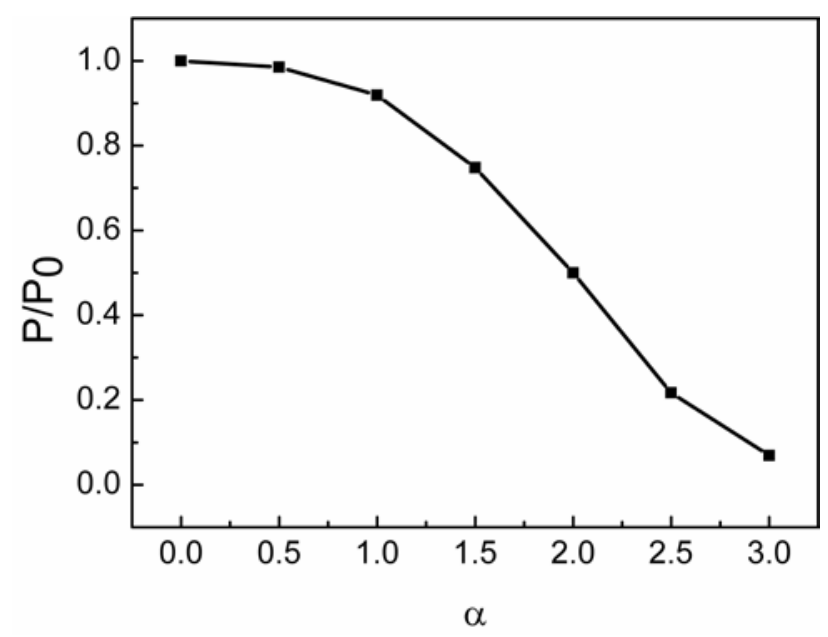

Fig. 3 The peak power ratio of the pump beam varies with truncation parameter.

Spatial spectrum distribution of the diffracted laser pulse which passes through a nonlinear medium with different truncation parameter is shown in Fig. 4. Spectrum distribution explains the reasons for the evolution of the new growth point. It is shown in Fig. 4 that the fastest growth frequency (arrow indicated) is away from the center frequency, which leads to the growth point increasingly far away from the center of beam. In addition, the spectral width increases with the decrease of truncation parameter. It is well known that spectral width decides the pulse width. So the pulse width would narrow with the decrease of truncation parameter. The temporal evolution of the pump beam in Fig.5 demonstrates this conclusion.

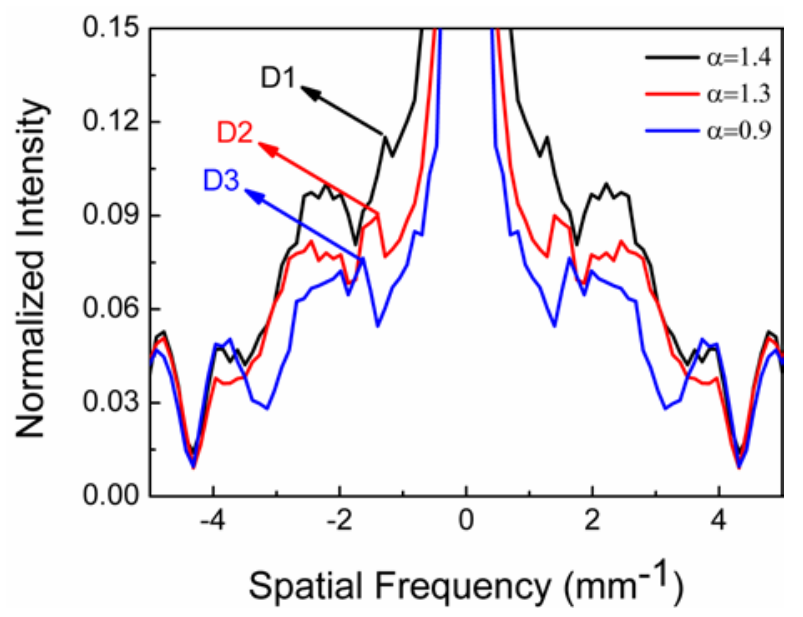

Fig. 4 The spatial spectrum distribution of SSSF varies with truncation parameter.

Fig. 5(a) shows the temporal distribution of the new growth point P1 for ultrashort laser pulse in different truncation parameter. Fig. 5(b) displays the pulse width varies with the truncation parameter. The pulse width decreases with the truncation parameter reducing. It is can be observed in Fig. 5 that the pulse would compress with the decrease of truncation parameter. The spatial intensity of growth point increases quickly with the decrease of truncation parameter. The rapid growth of the spatial intensity leads to strong nonlinear effect. The spectrum of the pulses is broadened by SPM and then the pulse width of the new growth point becomes narrow because of the changeable of spectrum. So the pulse width at the growth point P1 in space is compressed with the decrease of truncation parameter. 

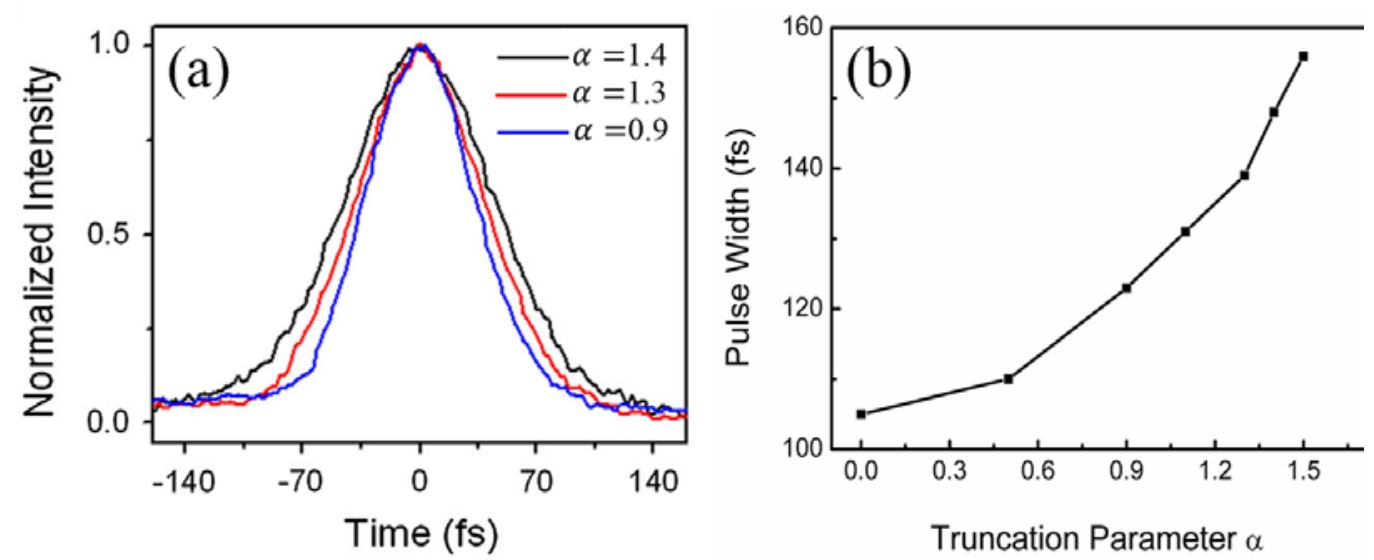

Fig. 5 (a) The temporal evolution of growth point varies with truncation parameter. (b) Pulse width as a function of different truncation parameter.

\section{Theoretical analysis for temporal evolution}

Ultrashort laser pulse is under a temporal and spatial interdependence during its propagation. The effect of SSSF enhances the intensity of the new growth point, which in turn affects the temporal evolution at this zone. Depending on the above analysis that the changeable of truncation parameter would change the actual peak power of the pump beam. The peak power would affect the strength of SPM, which would cause the spectrum to expand. And then the changeable of spectral width leads to the pulse compression and then the pulse width would decrease. In the experiment, the effect of dispersion can be neglected, since the dispersion effect traversing $5 \mathrm{~cm}$ of $\mathrm{CS}_{2}$ is the same with only changing the truncation parameter in the experiment. When an unchirped pulse is incident at $z=0$, the spectral broadening factor of the pump beam after propagation a distance $z$ in a nonlinear medium related to the input power by the relation[30]:

$$
\delta=\left(1+\frac{4}{3 \sqrt{3}} \phi_{\max }^{2}\right)^{\frac{1}{2}}
$$

Where $\phi_{\max }=\gamma P_{0} z, \gamma$ is nonlinear coefficient, $P_{0}$ is input power, $z$ is nonlinear propagation distance. The broadening factor is related to the input power and nonlinear propagation distance. As the input power increased, the spectral broadening factor increases.

The pulse width after propagation a distance $z$ in a nonlinear medium by the relation:

$$
T=T_{0} /\left(1+\frac{4}{3 \sqrt{3}} \phi_{\max }^{2}\right)^{\frac{1}{2}}
$$

Where $T_{0}$ is initial pulse width. According to the equation (3), the pulse width compresses with the increment of input power. The pulse width at P1 calculated according to equation (3) is shown in Fig. 6. 


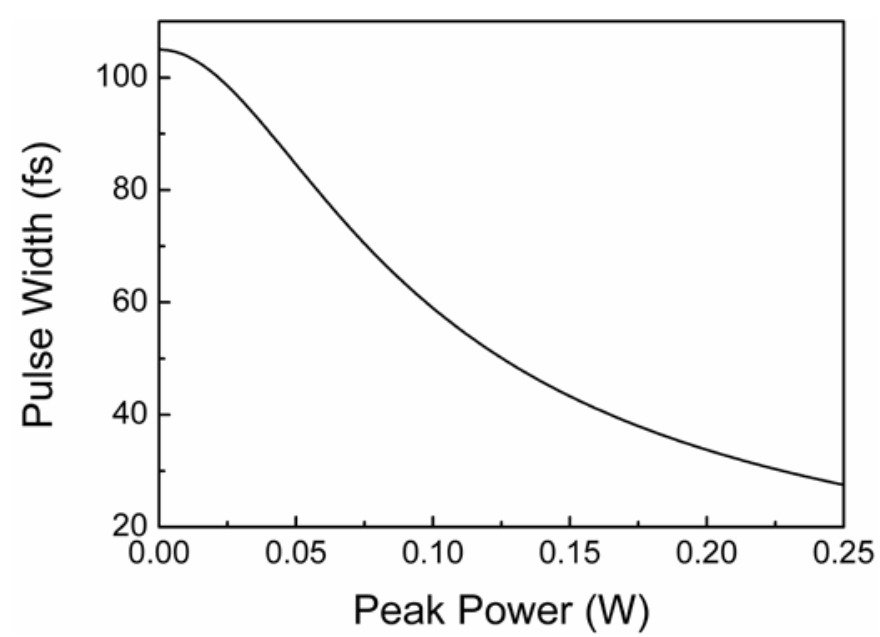

Fig. 6 Pulse width varies with peak power

It is illustrated in Fig. 6 that the pulse width decreases with the increment of input power. In the experiment, the input power changes with the truncation parameter. As the truncation parameter decreased, the peak power increases and the pulse width reduces. The calculated results are shown in Fig. 6, in agreement with the experimental results.

\section{Conclusions}

In summary, we demonstrate that SSSF can be created in $\mathrm{CS}_{2}$ by knife-edge diffraction. Spatial distribution of SSSF is determined by the truncation parameter of knife-edge diffraction. Our results indicate that when the truncation parameter is big, the influence of nonlinear effect is far less than the diffraction effect and the diffraction modulation plays a major role. As the truncation parameter decreased, the peak power increases which causes the effect of SPM to enhance and the off-axis energy to move towards the new growth point. It causes the spatial intensity of the new growth point to increase rapidly. We also obtain the spatial spectral distribution with different truncation parameter. We find the fastest growth frequency is increasingly far away from the center frequency and the spectral width increase in the same time. They causes the growth point to keep far away from the center of beam and the effect of SPM strengthen. Another, we analyze the effect of spatial modulation on pulse width. The peak power increases with the decrease of truncation parameter. The effect of SPM enhances with the increase of peak power. It leads to the spectral broadening factor large. The changeable of spectral width causes the pulse width to decrease. We also explain the evolution of spatial-temporal by theoretical analysis and the experimental results are agreement with the calculated results. We believe that an understanding of the detailed process of temporal evolutions at different partial spatial zones can help us to obtain a better understanding of the filaments of high power lasers.

\section{Acknowledgement}

This work was supported in part by the Specialized Research Fund for the Doctoral Program of Higher Education of China (20110161110012), the Department of Science and Technology of Hunan Province (2013FJ2018).

\section{References}

[1] Kandidov, V. P., Kosareva, O. G., Golubtsov, I. S., Liu, W., Becker, A., Akozbek, N. \& Chin, S. L., Self-transformation of a powerful femtosecond laser pulse into a white-light laser pulse in bulk optical media (or supercontinuum generation). Applied Physics B, 77(2-3), pp.149-165, 2003.

[2] Schweinsberg, A., Kuper, J., \& Boyd, R. W., Loss of spatial coherence and limiting of focal plane intensity by small-scale laser-beam filamentation. Physical Review A, 84(5), 053837, 2011. 
[3] Jia, H., Xu, B., Wang, F., \& Zhou, L., Small-scale self-focusing in a tapered optical beam. Applied optics, 51(25), pp.6089-6094, 2012.

[4] Chekalin, S. V., \& Kandidov, V. P., From self-focusing light beams to femtosecond laser pulse filamentation. Physics-Uspekhi, 56(2), 123, 2013.

[5] Deng, Y. B., Fu, X. Q., Tan, C., Yang, H., Deng, S. G., Xiong, C. X., \& Zhang, G. F., Experimental investigation of spatiotemporal evolution of femtosecond laser pulses during small-scale self-focusing. Applied Physics B, 114(3), pp.449-454, 2014.

[6] Chu, W., Li, G., Xie, H., Ni, J., Yao, J., Zeng, B. \& Xu, Z., A self-induced white light seeding laser in a femtosecond laser filament. Laser Physics Letters, 11(1), 015301, 2014.

[7] Dulkeith, E., Vlasov, Y. A., Chen, X., Panoiu, N. C., \& Osgood Jr, R. M., Self-phase-modulation in submicron silicon-on-insulator photonic wires. Optics express, 14(12), pp.5524-5534, 2006.

[8] Shim, B., Schrauth, S. E., \& Gaeta, A. L, Filamentation in air with ultrashort mid-infrared pulses. Optics express, 19(10), pp.9118-9126, 2011.

[9] Velchev, I., Pattnaik, R., \& Toulouse, J., Two-beam modulation instability in noninstantaneous nonlinear media. Physical review letters, 91(9), 093905, 2003.

[10] Canabarro, A. A., Santos, B., Gleria, I., Lyra, M. L., \& Sombra, A. S. B., Interplay of XPM and nonlinear response time in the modulational instability of copropagating optical pulses. JOSA B, 27(9), pp.1878-1885, 2010.

[11] Bespalov, V. I., \& Talanov, V. I., Filamentary structure of light beams in nonlinear liquids. ZhETF Pisma Redaktsiiu, 3, 471, 1966.

[12] Campillo, A. J., Shapiro, S. L., \& Suydam, B. R., Periodic breakup of optical beams due to self - focusing. Applied Physics Letters, 23(11), 628-630, 1973.

[13] Bliss, E. S., Speck, D. R., Holzrichter, J. F., Erkkila, J. H., \& Glass, A. J., Propagation of a high - intensity laser pulse with small - scale intensity modulation. Applied Physics Letters, 25(8), pp.448-450, 1974.

[14] Chekalin, S. V., \& Kandidov, V. P., From self-focusing light beams to femtosecond laser pulse filamentation. Physics-Uspekhi, 56(2), 123, 2013.

[15] Galvanauskas, A., High power fiber lasers. Optics and photonics news, 15(7), pp.42-47, 2004.

[16] Mironov, S., Lozhkarev, V., Luchinin, G., Shaykin, A., \& Khazanov, E., Suppression of small-scale self-focusing of high-intensity femtosecond radiation. Applied Physics B, 113(1), pp.147-151, 2013

[17] Semak, V. V., \& Shneider, M. N., Effect of power losses on self-focusing of high-intensity laser beam in gases. Journal of Physics D: Applied Physics, 46(18), 185502, 2013.

[18] Tan, C., Fu, X., \& Deng, Y., Experimental measurement of ultrashort pulse evolution at different spatial positions in nonlinear media. Optics \& Laser Technology, 59, pp.47-51, 2014.

[19] Dudley, J. M., Finot, C., Richardson, D. J., \& Millot, G., Self-similarity in ultrafast nonlinear optics. Nature Physics, 3(9), pp.597-603, 2007.

[20] Rohwetter, P., Queisser, M., Stelmaszczyk, K., Fechner, M., \& Wöste, L., Laser multiple filamentation control in air using a smooth phase mask. Physical Review A, 77(1), 013812, 2008.

[21] Cook, K., Kar, A., \& Lamb, R. A., White-light filaments induced by diffraction effects. Optics express, 13(6), pp.2025-2031, 2005.

[22] Gattass, R. R., \& Mazur, E., Femtosecond laser micromachining in transparent materials. Nature photonics, 2(4), pp.219-225, 2008. 
[23] Ross, I., Matousek, P., Towrie, M., Langley, A. J., \& Collier, J. L., The prospects for ultrashort pulse duration and ultrahigh intensity using optical parametric chirped pulse amplifiers. Optics Communications, 144(1), pp.125-133, 1997.

[24] Zozulya, A. A., Diddams, S. A., Van Engen, A. G., \& Clement, T. S., Propagation dynamics of intense femtosecond pulses: multiple splittings, coalescence, and continuum generation. Physical review letters, 82(7), 1430, 1999.

[25] La Fontaine, B., Vidal, F., Jiang, Z., Chien, C. Y., Comtois, D., Desparois, A., ... \& Mercure, H. P., Filamentation of ultrashort pulse laser beams resulting from their propagation over long distances in air. Physics of Plasmas (1994-present), 6(5), pp.1615-1621, 1999.

[26] Dachraoui, H., Oberer, C., Michelswirth, M., \& Heinzmann, U., Direct time-domain observation of laser pulse filaments in transparent media.Physical Review A, 82(4), 043820, 2010.

[27] Cambournac, C., Maillotte, H., Lantz, E., Dudley, J. M., \& Chauvet, M., Spatiotemporal behavior of periodic arrays of spatial solitons in a planar waveguide with relaxing Kerr nonlinearity. JOSA B, 19(3), pp.574-585, 2002.

[28] Akhmanov, S. A., Vorontsov, M. A., Ivanov, V. Y., Larichev, A. V., \& Zheleznykh, N. I., Controlling transverse-wave interactions in nonlinear optics: generation and interaction of spatiotemporal structures. JOSA B, 9(1), pp.78-90, 1992.

[29] Potasek M J. Modulation instability in an extended nonlinear Schrödinger equation. Optics letters, 12(11): pp.921-923, 1989.

[30] Agrawal G. P., Nonlinear fiber optics \& application of nonlinear fiber optics. Rochester, New York, 2006. 